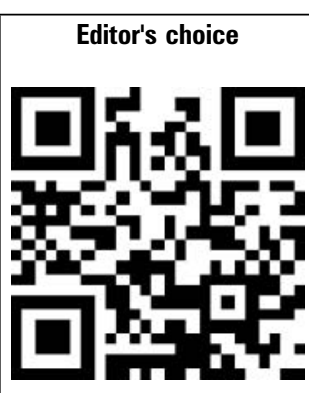

Scan to access more free content

Centre for Ophthalmology, University Eye Hospital Tuebingen, Eberhard-Karls University, Tuebingen, Germany

Correspondence to Dr M A Leitritz, University Eye Hospital, Centre for Ophthalmology, Tuebingen 72076, Germany; martinleitritz@googlemail.com

Received 29 April 2012 Revised 14 September 2012 Accepted 9 October 2012 Published Online First 15 November 2012
To cite: Leitritz MA, Gelisken F, Ziemssen F, et al. Br J Ophthalmol 2013;97:215-219.

\title{
Grid laser photocoagulation for macular oedema due to branch retinal vein occlusion in the age of bevacizumab? Results of a prospective study with crossover design
}

\author{
Martin A Leitritz, Faik Gelisken, Focke Ziemssen, Peter Szurman, \\ Karl U Bartz-Schmidt, Gesine B Jaissle
}

\section{ABSTRACT}

Background and aim To investigate the long term effectiveness of grid laser photocoagulation (GLP) versus intravitreal bevacizumab (BEV) in macular oedema (MO) secondary to branch retinal vein occlusion (BRVO), and to evaluate the treatment courses after treatments were switched.

Methods In this prospective interventional consecutive case series, previously untreated eyes with perfused MO were enclosed over a period of 16 months for BEV and for 29 months for GLP. The follow-up period was 1 year. Patients with persistent MO after 12 months of BEV were offered GLP and vice versa, and were followed-up for another 12 months.

Results Both BEV (23 eyes) and GLP (21 eyes) caused a significant $(p<0.05)$ reduction in central retinal thickness (CRT) at 12 months although this was delayed with GLP. However, BEV revealed a significantly better best corrected visual acuity (BCVA) compared with GLP (0.2 vs $0.5 \log M A R ; p<0.04)$. Switching therapy for non-responders revealed a reduced CRT at another 12 months, although this was not significant.

Conclusions Functionally and anatomically, BEV appears to be more effective than GLP for the therapy of MO due to BRVO. BCVA is significantly better after

1 year and the anatomical response of the $\mathrm{MO}$ is faster. Furthermore, non-responders with persistent $\mathrm{MO}$ despite BEV or GLP treatment might benefit from switching therapy.

\section{INTRODUCTION}

Branch retinal vein occlusion (BRVO) is the second most common retinal vascular disease after diabetic retinopathy. ${ }^{1}$ Development of chronic macular oedema (MO) is one of the main reasons for visual loss in BRVO. Grid laser photocoagulation (GLP) of the MO was the only evidence based treatment for years which had been approved in a controlled randomised clinical trial (in 1984). However, the study design showed relevant methodological problems, as only $83 \%$ and $62 \%$ of the eyes included completed the 2 and 3 years of follow-up, respectively. Moreover, the improvement in visual acuity was limited, requiring new therapies.

There are no systematic data to support surgical management, including vitrectomy with or without peeling of the inner limiting membrane, and sheathotomy. ${ }^{2-4}$ Application of triamcinolone acetonide or dexamethasone implants into the vitreous cavity was shown to be frequently associated with steroid induced adverse effects, in particular an increase in intraocular pressure and formation of cataract. ${ }^{5-7}$

A growing body of evidence has confirmed the important role of vascular endothelial growth factor in the pathogenesis of MO due to BRVO, suggesting therapy with ranibizumab (Lucentis, Genentech, San Francisco, California, USA) or bevacizumab (BEV; Avastin, Genentech). ${ }^{8-10}$ While ranibizumab was recently approved for the indication of MO after BRVO, the efficacy of BEV has only been described in smaller studies. In addition, one scientific evaluation directly compared BEV with GLP, but the results were impaired by relevant methodological shortcomings. ${ }^{11}$

Therefore, this study was designed to evaluate the effect of different pro re nata algorithms with GLP versus BEV in MO secondary to BRVO. The results of the GLP treatment arm can be regarded as an internal control, allowing for better interpretation of previously published BEV effects. ${ }^{12-14}$

Our prospective case series is the first to compare the long term effectiveness of intravitreal BEV versus GLP in BRVO induced $\mathrm{MO}$ which adheres to the frequent follow-up examinations, as well as the retreatment and inclusion criteria of the BRVO study. ${ }^{1}$ The results of the GLP arm can be regarded as an internal control, allowing direct comparison with the BRVO study. ${ }^{12-14}$ Moreover, this study investigated whether a switch in treatment modality for non-responders might be effective.

\section{PATIENTS AND METHODS}

\section{Inclusion criteria}

All patients with MO secondary to BRVO who met the inclusion criteria (box 1) according to the BRVO study ${ }^{1}$ were considered for enrolment in this prospective interventional case series. Consecutively, in the first 16 months (January 2006 to April 2007) patients were assigned to the BEV group ${ }^{13}$ and in the following 29 months (May 2007 to October 2009) patients were assigned to the GLP group (table 1). One patient in the GLP group was lost to follow-up before the first GLP treatment and therefore was excluded from the study due to missing baseline data. To ensure the efficacy of both treatment modalities, spontaneous resorption of intraretinal haemorrhage was awaited in all patients. The baseline examination included fluorescein angiography to assess the perfusion status of the macula. This investigation 


\section{Box 1 Interventional case series: inclusion criteria}

- Duration of branch retinal vein occlusion (BRVO) of 3-18 month

- Best corrected visual acuity of $20 / 40$ or less

- Perfused macular oedema

- No haemorrhage in the fovea

- No other disease that affects visual acuity

- No previous vitreoretinal surgery

- No previous laser treatment to the macular area

followed the tenets of the Declaration of Helsinki and was conducted in accordance with the local ethics committee.

In both groups, patients were followed-up for 48 weeks. The 1 year results of the BEV group have been published previously. ${ }^{13}$ Patients assigned to the BEV group were examined every 6 weeks and patients in the GLP arm were seen every 12 weeks. At each visit a complete examination, including best corrected visual acuity (BCVA) testing (ETDRS chart), slit lamp examination, dilated fundus examination and optical coherence tomography (OCT) were performed. Using OCT (Stratus OCT, Carl Zeiss Meditec Inc, Dublin, California, USA), central retinal thickness (CRT) was defined as the distance between the inner limiting membrane and the retinal pigment epithelium-choriocapillaris interface of radial lines through the foveal area. ${ }^{15}$ The fovea was determined using the patient's fixation and retinal landmarks. The callipers were set by hand as automated measurement protocols are more prone to error. ${ }^{16}$

\section{Intravitreal bevacizumab injection}

Briefly, intravitreal injections were performed according to the recommendations of the German Retina Society. ${ }^{17} \mathrm{BEV}$ was injected as $1.25 \mathrm{mg}$ in a $0.05 \mathrm{ml}$ total volume, applied via the pars plana. All BEV patients were informed about the off label use and the potential side effects. In signing an informed consent prior to the injections, patients confirmed that they were aware of the experimental nature of this therapy.

\section{Grid laser photocoagulation}

GLP was performed with an argon laser $(568 \mathrm{~nm}$; Coherent, Novus Omni, Santa Clara, California, USA) under topical anaesthesia by two senior operators. Laser spots were distributed over the area of angiographic leakage using a grid pattern. The major

Table 1 Characteristics of the subgroups

\begin{tabular}{|c|c|c|c|c|}
\hline Groups & $\begin{array}{l}\text { No of } \\
\text { patients }\end{array}$ & $\begin{array}{l}\text { Median age } \\
\text { at baseline } \\
\text { (years) } \\
\text { (min, max) }\end{array}$ & $\begin{array}{l}\text { Median duration } \\
\text { of BRVO (months) } \\
\text { (min, max) }\end{array}$ & $\begin{array}{l}\text { Sex } \\
\text { (M/F) }\end{array}$ \\
\hline \multicolumn{5}{|l|}{ Months 1-12 } \\
\hline 1st year BEV* & 23 & $68(45,80)$ & $7.1(3.0,16.6)$ & $14 / 9$ \\
\hline 1st year GLP & 21 & $70(56,84)$ & $7.4(2.9,18.5)$ & $11 / 14$ \\
\hline \multicolumn{5}{|l|}{ Months 12-24 } \\
\hline $\mathrm{BEV}>\mathrm{GLP}$ & 9 & $69(64,84)$ & $6.0(3.6,13.2)$ & $4 / 5$ \\
\hline $\mathrm{GLP}>\mathrm{BEV}$ & 6 & $68(50,72)$ & $6.4(4.5,16.4)$ & $4 / 2$ \\
\hline
\end{tabular}

vascular arcade defined the outer borders of the treated area, excluding the avascular zone. The spot size using Goldmann 3 Mirror Contact Lens was $50-100 \mu \mathrm{m}$. Laser intensity was adapted so that only tender effects were seen. Patients signed an informed consent prior to GLP therapy.

\section{Retreatment}

Retreatment was considered at each follow-up visit and performed after informed consent of the patient had been obtained, if $\mathrm{MO}$ on OCT in the foveal area still existed and BCVA was 20/32 or worse. BEV treatment was discontinued if, despite having three consecutive injections, BCVA did not increase by at least two lines or MO did not decrease by at least $30 \%$. The minimum time interval between GLP treatments was 6 months.

\section{Change of treatment}

Patients with persistent $\mathrm{MO}$ or no increase in BCVA after 12 months of treatment with BEV or GLP were offered a change of therapy to GLP or BEV, respectively. The therapy regimens were the same, as described above.

\section{Statistical analysis}

The main outcome measures of this investigation were BCVA and CRT, as measured by OCT. ETDRS letters were converted to $\log$ MAR units before analysis. The Wilcoxon signed rank and test and the Kruskal-Wallis test were performed to evaluate changes and homogeneity. Pearson analysis was performed to test for correlation (SPSS, V.18; SPSS Inc, Chicago, Illinois, USA). The significance level was set at $\alpha=0.05$.

\section{RESULTS}

This interventional case series prospectively enrolled 51 eyes from 51 consecutive patients with perfused $\mathrm{MO}$, according to the inclusion criteria (box 1). The time interval of inclusion was 16 months and 29 month for BEV and GLP, respectively. The first consecutive 26 patients underwent BEV injections (first year $\mathrm{BEV}$ ) and the following consecutive 25 patients underwent GLP (first year GLP). Patient characteristics are shown in table 1. Age and duration of $\mathrm{MO}$ were not significantly different between the two groups ( $\mathrm{p}=0.16$ and $\mathrm{p}=0.65$, respectively).

The 1 year results of the BEV treatment have been published previously. ${ }^{13}$ Briefly, 23 patients finished the 1 year follow-up (three patients were lost to follow-up and were excluded from the analysis: week 18, 18 and 36; final BCVA 20/32, 20/63 and 20/50; gain in BCVA lines $6,-1$ and 0 , respectively). The average number of injections was 3.4 (range 1-6). BEV resulted in a significant reduction in CRT, with median CRT decreasing from $395 \mu \mathrm{m}$ to $255 \mu \mathrm{m}$ by 12 months $(\mathrm{p}<0.05)$. Correspondingly, BCVA improved from a median BCVA of 0.50 $\log$ MAR at baseline to $0.2 \log$ MAR at 12 months $(p<0.001)$.

In the first year GLP group, 21 patients finished the 1 year follow-up. Four patients (16\%) were lost to follow-up at weeks 24, 36, 36 and 36 (final BCVA 20/50, 20/32, 20/100 and 20/ 40; gain in BCVA lines $0,2,2$ and 1 , respectively) and were excluded from the analysis. Ten visits were missed during the entire follow-up.

During the 12 month follow-up, a total of 46 GLP treatments were applied with an average number of 1.9 (range 1-3) per eye. The average interval between treatments was 33.1 weeks (range 23-65 weeks). The mean number of GLP spots was 45.6 (range 11-108). In the GLP group, the baseline median BCVA of $0.5 \log$ MAR improved to $0.45 \log$ MAR at 3 months $(\mathrm{p}<0.1)$ and increased significantly to a median BCVA of $0.45 \log$ MAR 
Figure 1 Boxplots showing the 12 month course of best corrected visual acuity (BCVA) $(A)$ and central retinal thickness (CRT) (B) in the first year grid laser photocoagulation group. Box and whisker diagrams show $5 \%$ and $95 \%$ quantiles (whiskers), $25 \%$ and $75 \%$ quantiles (box) and the median (marked by a line). Broken lines indicate median BCVA (A) and CRT (B) in the first year bevacizumab group, as published previously. ${ }^{13}$ This figure is only reproduced in colour in the online version.

at 6 months $(p<0.026)$. However, BCVA at 12 months $(0.5$ $\log M A R)$ showed no significant improvement compared with baseline $(\mathrm{p}<0.078)$ (figure 1A).

Comparison with BEV treatment revealed no significant difference in BCVA at baseline $(\mathrm{p}<0.57)$. However, at 3, 6, 9 and 12 months, BCVA in the BEV group was significantly better than that in the GLP group $(\mathrm{p}<0.041, \mathrm{p}<0.001, \mathrm{p}<0.017$ and $\mathrm{p}<0.04$, respectively). At 12 months, nine eyes $(42.8 \%)$ in the first year GLP group gained two or more lines whereas four eyes (19\%) lost two or more lines. In contrast, in the first year BEV group at 12 months, 18 eyes (69.2\%) improved two or more BCVA lines and no eye lost two or more lines. ${ }^{13}$

Compared with baseline (median $480 \mu \mathrm{m}$ ), in the first year GLP group there was a significant reduction in CRT at 3, 6, 9 and 12 months to a median of $413 \mu \mathrm{m}, 380 \mu \mathrm{m}, 375 \mu \mathrm{m}$ and $320 \mu \mathrm{m}$, respectively $(\mathrm{p}<0.03, \mathrm{p}<0.016, \quad \mathrm{p}<0.025$ and $\mathrm{p}<0.016$ ) (figure 1B). Comparison of CRT in the GLP and BEV groups revealed no significant difference, at baseline or at 3,6 , 9 or 12 months.

\section{Crossover of treatment modalities}

Change from bevacizumab treatment to grid laser

photocoagulation (BEV>GLP)

Of the first year BEV group, six patients received GLP treatment after 1 year of BEV therapy due to persistent $\mathrm{MO}$ and were followed-up for another 12 months. The average number of GLP treatments applied to this subgroup (BEV>GLP) during this 12 month follow-up interval was 1.7 (range 1-3) with an average time interval between treatments of 42.8 weeks (range 29-53 weeks).

Median BCVA was 0.40 logMAR before GLP treatment (12 months) and remained stable $(0.40$ logMAR $)$ until 24 months $(\mathrm{p}=0.85)$ (figure $2 \mathrm{~A})$. Initial median CRT was $445 \mu \mathrm{m}$ (12 months) and decreased to $340 \mu \mathrm{m}$ after 24 months, although this was not significant $(p=0.17)$ (figure $2 \mathrm{~B})$.
Change from grid laser photocoagulation to bevacizumab treatment (GLP>BEV)

Of the first year GLP group, nine patients received BEV treatment after 1 year of GLP therapy due to persistent MO and were followed for another 12 months. A total of 21 injections were applied to this subgroup (GLP>BEV) during this 12 month follow-up interval. The average number of injections was 2.3 (range 1-5) with an average time interval between treatments of 14.3 weeks (range 4-27).

Median BCVA was $0.60 \log$ MAR at 12 months, increasing to $0.50 \log$ MAR at 24 months $(\mathrm{p}=0.23)$ (figure $3 \mathrm{~A})$. Median CRT was $510 \mu \mathrm{m}$ at 12 months, decreasing to $430 \mu \mathrm{m}$ at 24 months $(\mathrm{p}=0.07)$ (figure $3 \mathrm{~B})$.

\section{Complications}

A lens touch due to the injection procedure was apparent in one eye (BCVA before lens touch $0.3 \log$ MAR; BCVA at 12 months $0.2 \log$ MAR). No cases of endophthalmitis, retinal detachment or any other severe procedure related or systemic complications were observed. Furthermore, no patient developed neovascular complications or needed peripheral sectorial laser photocoagulation during follow-up.

\section{DISCUSSION}

This prospective study is the first consecutive case series comparing GLP with BEV treatment for MO due to BRVO, with well defined retreatment criteria and constant follow-up examinations to prevent undertreatment. Comparable inclusion and treatment criteria in both groups confirmed the greater efficacy of BEV compared with GLP. ${ }^{11}$ There were no significant differences in the major demographics between the two groups (age, duration of BRVO, BCVA or CRT at baseline). The opportunity for direct comparison between the two treatment modalities and the crossover design may reveal why the functional outcome of GLP was worse. The crossover design is clearly inferior to a prospective randomised study due to potential
Figure 2 Bevacizumab>grid laser photocoagulation subgroup: course of best corrected visual acuity (BCVA) (A) and central retinal thickness (CRT) (B).
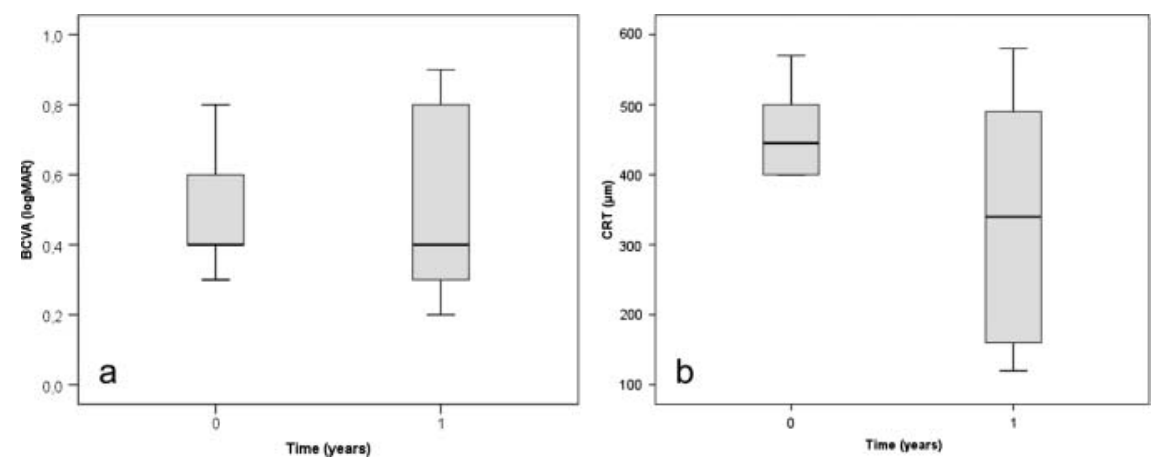
Figure 3 Grid laser

photocoagulation $>$ bevacizumab visual acuity $(B C V A)(A)$ and central retinal thickness (CRT) (B). subgroup: course of best corrected
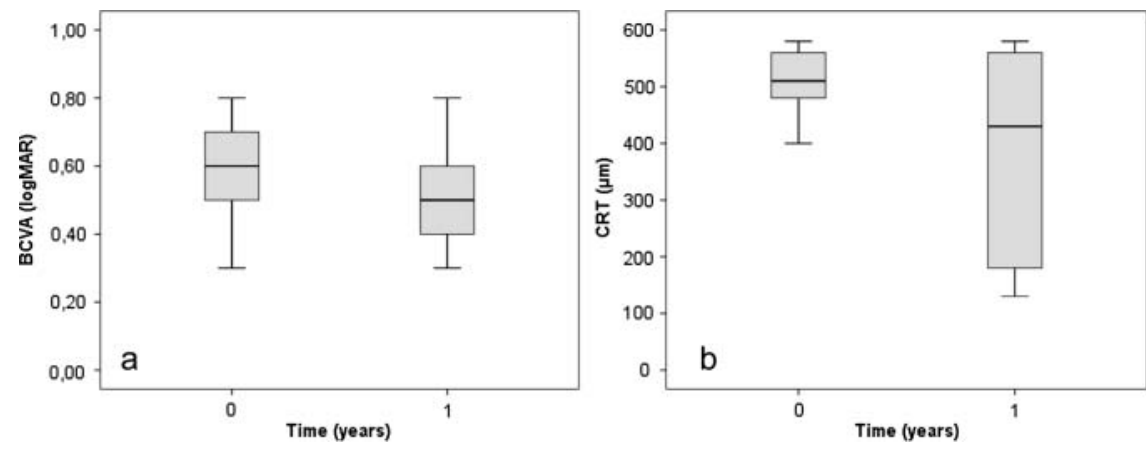

selection bias. Hence the results have to be discussed with caution.

BEV and GLP treatment resulted in a significant decrease in CRT, with no significant difference between the two groups. Contrasting with previous findings, ${ }^{11}$ the prominent finding of our study was the slower response in the GLP group (mean CRT $-14 \%$ at 3 months) compared with the BEV group (mean CRT $-27 \%$ at 3 months). Persistent MO may induce irreversible damage to the macula accompanied by persistent visual impairment. ${ }^{18}$ This is in accordance with our findings with GLP treatment, showing no significant increase in BCVA during the 12 month follow-up period. We assume that the delayed reduction in MO associated with GLP caused the significantly lower final visual outcome compared with the BEV group $(0.5$ $\log$ MAR and $0.25 \log$ MAR, respectively). The relevance of the duration of the MO was also noticed in other studies ${ }^{19}$ and can be derived from the CRUISE and GENEVA studies where deferred initiation of treatment (sham groups during the first 6 months) did not achieve the same extent of visual gain as seen after the primary treatment. ${ }^{20}$

The GLP group was designed as the control group, as the BRVO study has been the gold standard for the past few decades. Therefore, our investigation adhered strictly to the same inclusion criteria as the BRVO study to allow direct comparison. According to the non-logarithmic acuity steps used in the BRVO study, ${ }^{1}{ }^{13} 33.4 \%$ of our GLP group had an improvement of two or more BCVA lines at 12 months, comparable with the 1 year results of the BRVO study. ${ }^{1}$

An important question is treatment options for persistent $\mathrm{MO}$ despite BEV or GLP treatment. Therefore, this study investigated the efficacy of switching to the other treatment regimen for patients non-responsive to the initial treatment modality. Interestingly, stabilisation of BCVA in both groups during the 12 month interval was seen with a change in therapy. Moreover, switching the treatment modality was associated with a reduction of both the average number of injections (3.4-2.3 per year) and the average number of GLP treatments (1.9-1.7 per year). This is in accordance with a recent study of Donati et al, demonstrating a reduction in the number of intravitreal BEV re-injections in BRVO induced $\mathrm{MO}$ due to combined treatment with GLP. ${ }^{21}$ Another notable result of our study concerns the slight, although not significant, decrease in CRT in both groups following a switch in treatment modality. Taking into account the small sample number, it is not sufficient to identify this slight reduction as significant. Donati et al found a more pronounced, although not significant, decrease in CRT in a combined treatment modality of BEV and GLP compared with $\mathrm{BEV}$ alone. ${ }^{21}$ Ogino et al reported a substantial reducing effect on MO with a combined treatment of GLP and BEV in recurrent $\mathrm{MO}$, but only a limited effect on visual acuity. ${ }^{22}$ Therefore, based on these data, it is reasonable to assume that non- responders with persistent $\mathrm{MO}$ may benefit from a change in therapy or a combination therapy to limit macular damage due to chronic MO.

In conclusion, this study is the first to show the greater effectiveness of BEV compared with GLP using well defined retreatment criteria and frequent follow-up examinations. However, additional GLP appears to remain a treatment option for BEV non-responders.

Contributors All authors have contributed to the design and examinations performed in this study. All authors have contributed to the writing and reviewing of this manuscript in its current version.

Competing interests None.

Ethics approval The study was approved by the local ethics committee.

\section{REFERENCES}

1 Argon laser photocoagulation for macular edema in branch vein occlusion. The branch vein occlusion study group. Am J Ophthalmol 1984;98:271-82.

2 Mandelcorn MS, Nrusimhadevara RK. Internal limiting membrane peeling for decompression of macular edema in retinal vein occlusion: a report of 14 cases. Retina 2004:24:348-55.

3 Mester $U$, Dillinger $P$. Vitrectomy with arteriovenous decompression and internal limiting membrane dissection in branch retinal vein occlusion. Retina 2002;22:740-6

4 Weiss JN, Bynoe LA. Injection of tissue plasminogen activator into a branch retinal vein in eyes with central retinal vein occlusion. Ophthalmology 2001;108:2249-57.

5 Ramezani A, Esfandiari $H$, Entezari $M$, et al. Three intravitreal bevacizumab versus occlusion. Graefes Arch Clin Exp Ophthalmol 2012;250:1149-60.

6 Aref AA, Scott IU. Management of macular edema secondary to branch retinal vein occlusion: an evidence-based update. Adv Ther 2011;28:28-39.

7 Scott IU, Ip MS, VanVeldhuisen PC, et al. A randomized trial comparing the efficacy and safety of intravitreal triamcinolone with standard care to treat vision loss associated with macular edema secondary to branch retinal vein occlusion: the Standard Care vs Corticosteroid for Retinal Vein Occlusion (SCORE) study report 6. Arch Ophthalmol 2009:127:1115-28.

8 Guthoff R, Meigen T, Hennemann K, et al. Comparison of bevacizumab and triamcinolone for treatment of macular edema secondary to branch retinal vein occlusion in a pair-matched analysis. Ophthalmologica 2010;224:319-24.

9 Campochiaro PA, Heier JS, Feiner L, et al. Ranibizumab for macular edema following branch retinal vein occlusion: six-month primary end point results of a phase III study. Ophthalmology 2010;117:1102-12 e1.

10 Brown DM, Campochiaro PA, Bhisitkul RB, et al. Sustained benefits from ranibizumab for macular edema following branch retinal vein occlusion: 12-month outcomes of a phase III study. Ophthalmology 2011;118:1594-602.

11 Russo V, Barone A, Conte E, et al. Bevacizumab compared with macular laser grid photocoagulation for cystoid macular edema in branch retinal vein occlusion. Retina 2009;29:511-15.

12 Demir M, Oba E, Gulkilik G, et al. Intravitreal bevacizumab for macular edema due to branch retinal vein occlusion: 12-month results. Clin Ophthalmol 2011;5:745-9.

13 Jaissle GB, Leitritz M, Gelisken F, et al. One-year results after intravitreal bevacizumab therapy for macular edema secondary to branch retinal vein occlusion. Graefes Arch Clin Exp Ophthalmol 2009;247:27-33.

14 Rogers SL, McIntosh RL, Lim L, et al. Natural history of branch retinal vein occlusion: an evidence-based systematic review. Ophthalmology 2010:117:1094-101 e5.
Provenance and peer review Not commissioned; externally peer reviewed. two intravitreal triamcinolone injections in recent-onset branch retinal vein 
15 Bolz M, Ritter M, Polak K, et al. (The role of stratus OCT in anti-VEGF therapy. Qualitative and quantitative assessment of neovascular AMD). Ophthalmologe 2008:105:650-5.

16 Sadda SR, Wu Z, Walsh AC, et al. Errors in retinal thickness measurements obtained by optical coherence tomography. Ophthalmology 2006;113:285-93.

17 Jaissle GB, Szurman P, Bartz-Schmidt KU. (Recommendation for the implementation of intravitreal injections-statement of the German Retina Society, the German Society of Ophthalmology (DOG) and the German Professional Association of Ophthalmologists (BVA)). Klin Monbl Augenheilkd 2005;222:390-5.

18 Noma $\mathrm{H}$, Funatsu $\mathrm{H}$, Yamasaki $\mathrm{M}$, et al. Aqueous humour levels of cytokines are correlated to vitreous levels and severity of macular oedema in branch retinal vein occlusion. Eye (Lond) 2008:22:42-8.
19 Ehlers JP, Decroos FC, Fekrat $\mathrm{S}$. Intravitreal bevacizumab for macular edema secondary to branch retinal vein occlusion. Retina 2011;31: $1856-62$.

20 Yeh WS, Haller JA, Lanzetta P, et al. Effect of the duration of macular edema on clinical outcomes in retinal vein occlusion treated with dexamethasone intravitreal implant. Ophthalmology 2012;119:1190-8

21 Donati S, Barosi $\mathrm{P}$, Bianchi M, et al. Combined intravitreal bevacizumab and grid laser photocoagulation for macular edema secondary to branch retinal vein occlusion. Eur J Ophthalmol 2011;22:607-14.

22 Ogino K, Tsujjkawa A, Murakami T, et al. Grid photocoagulation combined with intravitreal bevacizumab for recurrent macular edema associated with retinal vein occlusion. Clin Ophthalmol 2011;5:1031-6. 